\title{
CDISC SDTM Not Done Terminology
}

National Cancer Institute

\section{Source}

National Cancer Institute. CDISC SDTM Not Done Terminology. NCI Thesaurus. Code C66789.

Terminology codelist used with Not Done within the Clinical Data Interchange Standards Consortium Study Data Tabulation Model. 